\title{
Effect of a Digital Precision Public Health Intervention for Care Coordination During Covid-19 Pandemic in Australia: A Real World Study
}

Andre Q Andrade ( $\square$ andre.andrade@unisa.edu.au )

University of South Australia https://orcid.org/0000-0001-6587-3169

Jean-Pierre Calabretto

University of South Australia

Nicole L Pratt

University of South Australia

Lisa M Kalisch-Ellett

University of South Australia

Vanessa T LeBlanc

University of South Australia

Elizabeth E Roughead

University of South Australia

\section{Research}

Keywords: COVID-19, pandemic, severe infection, national emergencies, public health initiatives

Posted Date: September 17th, 2021

DOI: https://doi.org/10.21203/rs.3.rs-882615/v1

License: (c) (1) This work is licensed under a Creative Commons Attribution 4.0 International License.

Read Full License 


\section{Abstract}

Background: Emergencies disproportionally affect vulnerable populations. The COVID-19 pandemic affected older patients with co-morbidities both directly due to more severe infection and indirectly by affecting care provision. To promote continuity of care, public health professionals require tools to quickly and precisely coordinate with primary care providers. This study evaluated whether digital interventions powered by current existing infrastructure are more effective than conventional interventions in promoting primary care appointments during the COVID-19 pandemic.

Methods: We developed a digital intervention delivered by secure messaging and compared it to a post delivered intervention to promote continuity of care for vulnerable veterans during COVID-19 in a real world, non-randomised, interventional study. The study was implemented as part of the Veterans' MATES program, an Australian Government Department of Veterans' Affairs program to promote improvements in health care for veterans. The intervention provided patient specific information to general practitioners (GPs) to support continuity of care, alongside mailed education to veterans. The intervention key messages were to maintain regular contact with care providers and to continue to adhere to health plans. The intervention took place in April 2020, during the first weeks of COVID-19 social distancing rules in Australia. The main outcome was time to first appointment with the primary general practitioner (GP) measured using a Cox proportional hazards model.

Results: GPs received digital messaging for 51,052 veterans and post messaging for 26,859 veterans. The proportion of patients seeing their primary GP during the three months following intervention was higher in the digital group $(77.8 \%)$ than the post group $(61.5 \%)(p<0.01)$. Being in the digital group was associated with earlier appointments.

Conclusion: Current infrastructure coupled with innovative solutions is effective in promoting care coordination at scale during national emergencies, opening up new perspectives for precision public health initiatives.

\section{Contributions To The Literature}

- Care disruptions due to national emergencies, such as the COVID-19, have a long-lasting impact on vulnerable individuals. We describe a real world, countrywide digital intervention to promote continuity of care.

- We demonstrated the capacity of a digital intervention to identify vulnerable individuals, reach and engage their main care providers, and measure outcomes. The digital intervention was more effective than a postal intervention at promoting early primary provider visits.

- We envisage such systems used to guarantee the supply of medicines use for chronic diseases, promote support for vulnerable patients, and maintain care for persons with care-time critical illnesses. 


\section{Background}

There is significant evidence that national emergencies, such as natural disasters or pandemics, result in long-lasting health consequences, including increased mortality months after the initial event [1]. The consequences disproportionally affect vulnerable populations, such as older people, the poor and people with mental illness [2]. The COVID-19 pandemic is an example of a harmful national emergency. It is estimated that current disruptions in health care due to COVID-19 will cause post-pandemic increases in child mortality by up to $44 \%$ in low and middle income countries [3].

The COVID-19 pandemic affects older individuals with at least one co-morbidity in three different ways. Firstly, the infection is more severe in this population leading to more hospitalisations [4] and higher fatality rate [5]. Secondly, the changes induced by the pandemic and its prevention may increase the prevalence of co-morbidities, such as mental conditions [6]. The third impact of national emergencies is the interruption in service provision [7], with potential for reduced number of face-to-face attendances, hospital visits and laboratory tests.

As a response to the COVID-19 pandemic, the Australian government implemented a series of policies to reduce the risk of widespread infection. Starting on March 2nd 2020, the policies included stay at home recommendations where possible for persons at high risk of poor outcomes if they were to develop COVID-19. To prevent disruptions, the Australian government also implemented a national health plan to maintain access to health services during the pandemic, including options for many medical attendances to be provided by video or telehealth where appropriate. Video and telehealth Medicare items were available for persons at risk of health care harms from COVID-19 and in quarantine from March 13th 2020.[8] Telehealth services were extended to enable vulnerable medical practitioners and health practitioners to provide telehealth for all their patients from March 23rd 2020, and further expanded to all practitioners and all patients from March 29th 2020.[9] Major social distancing restrictions came into effect, including work from home where possible, from the week of March 23rd 2020 onwards. Despite implementation of video and telehealth options, there were concerns that many people may have delayed or avoided their health care appointments due to concerns about catching the virus or concerns about not wanting to overload busy doctors. Nationally, a 10\% reduction was observed in Medicare Services in April 2020 compared to April 2019 [10].

Given the importance of continuous care provision for patients with chronic diseases, it is the responsibility of public health professionals to plan and promote strategies to ensure that the needs of patients and caregivers are addressed [11]. One of the key aspects of care coordination is to identify vulnerable individuals and advise them and their care providers, triggering appropriate action. In this paper, we describe a real world, large scale intervention as a rapid response to the COVID-19 pandemic. This initiative was developed as part of the Veterans' MATES program. The Veterans' Medicines Advice and Therapeutics Education Services (Veterans' MATES) program is an Australian Government Department of Veterans' Affairs (DVA) funded program aimed at improving medicine and health services use and health outcomes for all persons in the veteran community across Australia. 


\section{Methods}

\section{Context and setting}

In Australia, the General Practitioner (GP) is the cornerstone of primary care coordination. About $84 \%$ of Australians see a GP every year, and $77 \%$ of patients have a preferred GP [12]. The goal of the proposed intervention was to help GPs identify their vulnerable patients and promote follow up appointments during the period of restrictions. Some particularities of the Australian health system determined the technology choice:

- Geographical location - Veterans are distributed across Australian states and territories. While there are a few GPs specialised in veteran care, most have less than 4 veterans under their care. Patients are free to choose their GP irrespective of geographical location. While this may increase patient satisfaction and access, the lack of patient registration makes it harder for practices to define their population, potentially reducing continuity of care [13].

- Technological readiness - Australian GPs have had near universal use of electronic health records for more than 10 years [14] and a large penetration of secure messaging infrastructure for receiving laboratory test results.

- Public funded, privately operated model - Due to the health model in Australia, any intervention focused on GPs must be highly collaborative and involve practitioners from the start. There is a high degree of agency during GP appointments, and payers have limited influence on practice.

\section{The Veterans' MATES program}

The Veterans' MATES program is a multifaceted intervention, composed of an educational component and an audit and feedback component delivered to general practitioners (GPs), supported by educational components delivered to pharmacists, other relevant healthcare professionals and veterans. Interventions are created in three sequential steps.

The first step is an epidemiological inquiry to identify trends and potential issues in healthcare access and use. Examples include: long term prescription of medicines recommended for acute issues; doses above guideline recommendations; and lack of screening tests for an eligible population. The program has access to the DVA health claims database, updated monthly. This database includes all dispensed medicines requiring prescription, claimed healthcare services and laboratory services, home care and aged care.

The second step is the development of educational material and audit and feedback documents. This is a collaborative process with heavy stakeholder involvement, including multiple health professionals and behaviour change specialists. 
The final step is the identification and delivery of the intervention to veterans and their main healthcare provider. This step requires use of patient-level information contained in the database to print and post via regular mail personalised audit and feedback documents at scale, reaching tens of thousands of veterans and GPs per intervention.

The program has been extensively described elsewhere [15]. It has been shown to be effective for changing professional behaviour in different domains [15], including promoting medicine review [16], osteoporosis screening [17], uptake of health services [18], reducing inappropriate proton pump inhibitor use [19] and hypnotic use for insomnia [20].

The Veterans' MATES program ran the first intervention in 2004 and, since then, has delivered 4 interventions each year. In 2019, a digital precision public health initiative was started to make the best use of digital technology infrastructure and replace all printed materials. The proposed solution was primarily aimed at improving intervention efficacy by increasing GP engagement and reducing the lag between the detection of an issue and its notification to the GP.

\section{Study design and sample}

To evaluate the effectiveness of a digital precision public health intervention in promoting continuity of care during national emergencies, we performed a non-randomised experimental study. As part of the Veterans' MATES program, we developed a digital intervention to promote care provision for patients vulnerable to poor outcomes following COVID-19 infection compared to usual care (paper based intervention, sent by post).

Patient allocation to postal or digital group was done in two sequential steps. First, eligible patients were identified based on information contained in the administrative health claims database. Identification of vulnerable individuals was based on the best information at the time, and focused on the population at highest risk of poor outcomes from COVID-19, which was persons aged over 70 years with the following comorbidities, hypertension [21-28], chronic heart disease [21-28], diabetes [21-29], chronic airways disease [22-28], cerebrovascular disease [21, 22, 25, 30] , chronic liver disease [27] , chronic renal failure $[22,24,26-28]$, malignancy $[21,22,25,26,28,31]$ or being immunocompromised [27]. Identification algorithms were composed of clinical rules with varying levels of complexity, looking for past diagnostic codes (ICD-10) during hospitalisations, use of medicines indicating treatment for one of the target comorbidities (e.g., chronic carvedilol use in heart failure), and combinations of services and medicines used.

After patient eligibility checking, the primary GP was identified using a proprietary algorithm based on the frequency and recency of appointments. General practitioners with at least one eligible patient were eligible for the intervention. All GPs who had the capability to receive the digital intervention (access to electronic health record and secure message delivery) were included in the digital group. The remainder was included in the post group. 


\section{Intervention development}

The main goal of this intervention was to promote continuity of care during lockdown and social restrictions. The underlying theory was that the provision of personalised recommendations in the form of an audit and feedback document delivered directly to a GP's clinical software would influence patient recall and trigger an early appointment. The initiative was conducted using a collaborative, pragmatic approach, influenced by Greenhalgh et al diffusion of innovations model [32], in order to develop a solution that could be implemented at national scale. The model summarises a collection of theoretical and empirical findings, and highlights the interplay between an innovation, the adopter, the context in which the innovation takes place, the implementation and the diffusion process. The model suggests innovation developers to consider 9 dimensions during intervention creation: 1) Innovation; 2) Adopter; 3 ) Assimilation; 4) Communication and Influence; 5) System Antecedents for Innovation; 6) System Readiness for Innovation; 7) Outer Context; 8) Implementation Process; 9) Linkage.

The processes involved in intervention development complements the 3-steps used by Veterans' MATES interventions, suited for rapid care coordination (see Figure 1). The development of all content and interventions is based on the best evidence available at the time and supported by repeated reviews by healthcare professional panels. The audit and feedback document was developed and submitted to a stakeholder review group, including health professionals (pharmacists, GPs, among others). The behaviour change techniques used included goal setting (e.g., "Schedule appointments to ensure vulnerable patients are still receiving necessary care"), prompts (e.g., medicine dispensing suggesting respiratory disease), and information about health consequences (e.g., rationale for recommendations). A sample can be seen in Figure 2.

All veterans living in the community $(68,872$ individuals) received educational material delivered by post about COVID-19 infection prevention prompting them to maintain regular contact with care providers and to continue to adhere to health plans, as well as how to access care during the pandemic given the expansion of video and telehealth appointments and free medicine delivery services for eligible persons. The online version of the printed materials can be seen in [33].

\section{Outcomes and statistical analysis}

The main outcome of the intervention was the time to first appointment with the primary GP. Australia had in place restrictions on visitors to aged care, thus, we excluded veterans living in aged care from the time-to-event analysis. To compare the digital and the postal groups, we performed a time-to-event analysis, considering the first visit to the primary GP since the intervention delivery date as the target event. Determinants of an early GP appointment were analysed using Cox regression. The analysed variables included age at the time of intervention, gender and number of appointments in the previous year (April/2019 to March/2020). 
Given the large sample and the purposeful sampling, we considered a $99 \%$ confidence interval $(p<=0.01)$ for all hypothesis tests. All analyses were performed in Python 3.7. The main statistical library used for time-to-event was lifelines 0.25 [34].

\section{Results}

A total of 77,911 veterans were targeted for the intervention, and 18,577 GPs were identified as their main care provider. Among those GPs, $61.2 \%(11,375)$ were eligible to receive secure message documents. The 51,052 veterans who had these GPs as their main care providers were included in the digital group. The remaining 7,202 GPs were not eligible for digital delivery, and the 26,859 veterans under their care were included in the post group. Veterans in both groups were similar in age, gender and geographical distribution (Table 1). However, patients assigned to the digital group had a higher number of visits with the primary GP during 2019 (prior to COVID-19 pandemic), despite a similar profile in number of comorbidities.

A total of 51,052 individualised messages were sent to GPs in four sequential batches, starting on 29/Apr/2020 over secure message. A remainder of 26,859 messages were posted to GPs ineligible for secure message delivery. From the cohort of 51,052 veterans and their GPs targeted for secure message, we received $4(<0.01 \%)$ messages from users advising that they were not the primary care provider of targeted patient, suggesting the algorithm for finding the primary GP was highly accurate.

The total number of appointments with GPs increased substantially over April/2020, from 20,425 visits in the last week of March (25/03 to 31/03) to 25,214 in the last week of April (22/04 to 28/04). The appointment numbers increased with both the primary GP or other GPs, and was largely driven by services provided by telehealth. (see Figure 3).

\section{Time-to-event analysis}

We found that most targeted veterans had at least one appointment with their primary GP $(72.3 \%)$ during the three months after intervention. The proportion of patients seeing their primary GP was higher in the digital group $(77.8 \%)$ than the post group $(61.5 \%),(p<0.01)$ which is reflected in the Kaplan-Meier curve (see Figure 4).

To account for the possible influence of the number of GP visits in the prior year, we included the number of appointments in the prior year as an interaction factor (see Table 2). After adjustment, the digital delivery still had a statistically significant influence on the chance of early GP visit with the primary provider.

We stratified by 1) group and number of appointments in 2019; and 2) group and number of comorbidities (see Figure 5). For number of appointment in 2019, delivery through the digital medium still 
increased the chance of having a primary provider visit during the three months following the intervention when compared to post.

\section{Discussion}

This study shows successful use of current infrastructure to promote care coordination at a national level during emergencies. We demonstrated the capacity of a digital intervention to identify vulnerable individuals, reach and engage their main care providers, and measure outcomes. Additionally, we found that the digital intervention was more effective than a post intervention at promoting early primary provider visits.

Adequate infrastructure coupled with innovative solutions enables the promotion of care coordination at scale during national emergencies, opening new perspectives for precision public health initiatives. The results suggest that solutions using current digital infrastructure can be allies in emergency preparedness systems. Experiences after national emergencies and disasters recognise their impact on patients with chronic diseases and the importance of quickly reacting to healthcare needs of these patients when designing plans $[35,36]$. Given that responses can vary according to emergency and condition, the capacity to identify patients with particular comorbidities may prevent consequences on vulnerable patients, such as cancer patients [37] and patients with severe kidney disease [38]. Our study shows innovative methods of data analysis can be used to extract signals from administrative claims database, in particular those containing therapeutic information (medicines and services). We further showed secure messaging infrastructure can be used to quickly reach primary providers, and behavioural theory influenced digital interventions are effective in promoting care continuity by the primary provider. We envisage such systems being used to guarantee the supply of medicines use for chronic diseases, promote support for patients with mental health conditions, maintain care for persons with care-time critical illnesses, such as persons in active bouts of chemotherapy, persons undergoing dialysis, persons requiring home delivered oxygen, and maintain care of elderly patients.

An interesting and unexpected finding was the difference in number of appointments between the digital and post group. The only criteria separating both groups was the access to secure message delivery. Since no other differences between groups were identified, it is possible that the access to technology itself is promoting continuity of care. However, as shown by the Cox regression and the partial effect plots, the digital intervention retained its effect even after adjusting for the number of appointments in the previous year. Both findings suggest that access to technology and secure message delivery should be promoted to clinicians as ways to promote continuity of care.

This study reinforces the value of developing solutions fit for context using iterative and participatory processes. Finding the right level of complexity of any digital health intervention is particularly susceptible to context change. We profited from the existence of a mature claims databases, which are routinely updated and contain useful clinical information. We also took advantage of secure messaging infrastructure, which was already incorporated into the clinician workflow. Finally, the existence of 
standards (HL7 and pdf) and availability of structured computer coding libraries provided the required flexibility, speed and power to develop the intervention. We also profited from having strong stakeholder relationships having stakeholder reference groups that had continuously met since program inception in 2004. The time from conception to full implementation was less than 4 weeks, which included required approvals and having clinical and stakeholder review prior to implementation. The stakeholder and clinical goodwill that had been achieved due to engagement with Veterans' MATES over time, meant that a team of people were available to review materials at short notice, including on the ground practitioners who felt they were being bombarded with information in the early days of the pandemic.

This study benefited from a well established program, describing the results of a large scale intervention at national level. The large sample size increases the confidence in the results and allowed the stratification of distinct covariate effect in promoting early GP visits.

The main limitation was the lack of a randomised controlled environment to isolate the effects of the intervention alone in promoting GP visits for vulnerable individuals. The urgency of the situation and the current system capacity were the paramount issues driving the study design. The comparison with the post group may have been biased by the purposeful sampling, despite adjusting for the number of usual GP visits in a year. Several policies were enacted at different times of the COVID-19 pandemic, which may have influenced the results. These include Policy changes made by the Australian Department of Health, which promoted the use of telehealth by all Australians (including veterans). Telehealth ensured continued access to providers despite social restrictions and was sustained throughout the study period. It is possible our digital group were better set up for telehealth provision. Furthermore, this intervention took place in the Australian health context, which may have influenced early adoption of digital solutions. Different health systems will need to adapt to local technologies and capabilities.

\section{Conclusion}

This study is one of the first to show the feasibility and increased efficacy of a digital intervention to coordinate care at national level during emergencies Digital delivery (via secure message infrastructure) promoted visits to the primary GP. Similar solutions can be adapted as a response to emergency events to ensure care continuation of vulnerable populations.

\section{Declarations}

\section{Ethics approval and consent to participate}

An ethics protocol for the study was approved by the University of South Australia Human Research Ethics Committee (ethics protocol P203/04) and the Department of Veterans' Affairs Human Research Ethics Committee (E016/007).

\section{Consent for publication}




\section{Availability of data and material}

The data that support the findings of this study are available from the Australian Government Department of Veterans' Affairs but restrictions apply to the availability of these data, which were used under license for the current study, and so are not publicly available.

\section{Competing interests}

The authors have no competing interests to declare.

\section{Funding}

This work was funded by the Australian Government Department of Veterans' Affairs (DVA) as part of the Veterans' Medicines Advice and Therapeutics Education Services (Veterans' MATES) program. EER is supported by the National Health and Medical Research Council (GNT 1110139). DVA reviewed this manuscript before submission but played no role in study design, execution, analysis or interpretation of data, writing of manuscript or decision to submit the paper for publication.

\section{Authors' contributions}

AQA conceived of, designed, conducted the final analysis for this paper and drafted the manuscript. AQA, J-PC, VTL and EER developed the protocol and study approach. LMKE and NLP were involved in the data analysis. EER conceived of and designed the study, and critically revised the manuscript for important intellectual content. All authors made important contributions to the theoretical approach and interpreting insights. All authors read and approved the final manuscript.

\section{Acknowledgements}

The research was funded by the Australian Government Department of Veterans' Affairs as part of the delivery of the Veterans' Medicines Advice and Therapeutics Education Services (Veterans' MATES) program. Veterans' MATES is provided by the University of South Australia, Quality Use of Medicines and Pharmacy Research Centre, in association with Discipline of General Practice, The University of Adelaide; Discipline of Public Health, The University of Adelaide; Repatriation General Hospital, Daw Park; NPS Better choices, Better health; Australian Medicines Handbook; and the Drug and Therapeutics Information Service. 


\section{References}

1. Santos-Burgoa C, Sandberg J, Suárez E, Goldman-Hawes A, Zeger S, Garcia-Meza A, et al. Differential and persistent risk of excess mortality from Hurricane Maria in Puerto Rico: a time-series analysis. The Lancet Planetary Health. 2018 2018/11/01/;2(11):e478-e88. doi: https://doi.org/10.1016/S2542-5196(18)30209-2.

2. Dempsey TM, Lapinsky SC, Melnychuk E, Lapinsky SE, Reed MJ, Niven AS. Special Populations: Disaster Care Considerations in Chronically III, Pregnant, and Morbidly Obese Patients. Crit Care Clin. 2019 Oct;35(4):677-95. doi:10.1016/j.ccc.2019.06.010. PMID: 31445613.

3. Roberton T, Carter ED, Chou VB, Stegmuller AR, Jackson BD, Tam Y, et al. Early estimates of the indirect effects of the COVID-19 pandemic on maternal and child mortality in low-income and middleincome countries: a modelling study. The Lancet Global Health. 2020;8(7):e901-e8. doi:10.1016/S2214-109X(20)30229-1.

4. Bajgain KT, Badal S, Bajgain BB, Santana MJ. Prevalence of comorbidities among individuals with COVID-19: A rapid review of current literature. American Journal of Infection Control. 2020 2020/07/10/. doi: https://doi.org/10.1016/j.ajic.2020.06.213.

5. Harrison SL, Fazio-Eynullayeva E, Lane DA, Underhill P, Lip GYH. Comorbidities associated with mortality in 31,461 adults with COVID-19 in the United States: A federated electronic medical record analysis. PLOS Medicine. 2020;17(9):e1003321. doi:10.1371/journal.pmed.1003321.

6. Fisher JR, Tran TD, Hammarberg K, Sastry J, Nguyen H, Rowe H, et al. Mental health of people in Australia in the first month of COVID-19 restrictions: a national survey. 2020;213(10):458-64. doi: https://doi.org/10.5694/mja2.50831.

7. Czeisler ME, Marynak K, Clarke KEN, Salah Z, Shakya I, Thierry JAM, et al. Delay or Avoidance of Medical Care Because of COVID-19-Related Concerns - United States, June 2020. Morbidity and Mortality Weekly Report. 2020 9/11;69(36):1250-7. PMID: SCOPUS:85090880129. doi: 10.15585/mmwr.mm6936a4.

8. Health AGDo. Coronavirus (COVID-19) health package announced. Canberra: Commonwealth of Australia; 2020 [cited 20201 June]; Available from: https://www.health.gov.au/news/coronaviruscovid-19-health-package-announced.

9. Australian Government Department of Health. $\$ 1.1$ billion to support more mental health, Medicare and domestic violence services. Canberra: Commonwealth of Australia; 2020 [cited 20201 June ]; Available from: https://www.health.gov.au/news/11-billion-to-support-more-mental-health-medicareand-domestic-violence-services .

10. Health, Alo. Welfare. Impacts of COVID-19 on Medicare Benefits Scheme and Pharmaceutical Benefits Scheme service use. Canberra: AlHW; 2020.

11. Kluge HHP, Wickramasinghe K, Rippin HL, Mendes R, Peters DH, Kontsevaya A, et al. Prevention and control of non-communicable diseases in the COVID-19 response. Lancet (London, England). 2020 May 30;395(10238):1678-80. PMID: 32401713. doi: 10.1016/s0140-6736(20)31067-9. 
12. The Royal Australian College of General Practitioners. General Practice: Health of the Nation 2019. East Melbourne: RACGP; 2019.

13. Taylor CJ, Wright M, Jackson CL, Hobbs R. Grass is greener? General practice in England and Australia. Br J Gen Pract. 2016;66(649):428-9. doi:10.3399/bjgp16X686377. PMID: 27481974.

14. Jha AK, Doolan D, Grandt D, Scott T, Bates DW. The use of health information technology in seven nations. Int J Med Inform. 2008 Dec;77(12):848-54. PMID: 18657471. doi:

10.1016/j.ijmedinf.2008.06.007.

15. Roughead EE, Kalisch Ellett LM, Ramsay EN, Pratt NL, Barratt JD, LeBlanc VT, et al. Bridging evidence-practice gaps: improving use of medicines in elderly Australian veterans. BMC health services research. 2013 Dec 12;13:514. PMID: 24330781. doi: 10.1186/1472-6963-13-514.

16. Kalisch Ellett LM, Pratt NL, Sluggett JK, Ramsay EN, Kerr M, LeBlanc VT, et al. Sustaining practice change in health care: the impact of a national quality improvement program on the uptake of collaborative medicines reviews. Journal of Pharmacy Practice Research. 2018;48(3):222-30. doi:10.1002/jppr.1379.

17. Kalisch Ellett LM, Pratt NL, Sluggett JK, Ramsay EN, Kerr M, LeBlanc VT, et al. Patient-specific prescriber feedback can increase the rate of osteoporosis screening and treatment: results from two national interventions. Archives of osteoporosis. 2017 Dec;12(1):17. PMID: 28188561. doi: 10.1007/s11657-017-0309-4.

18. Pratt NL, Kalisch Ellett LM, Sluggett JK, Ramsay EN, Kerr M, LeBlanc VT, et al. Commitment questions targeting patients promotes uptake of under-used health services: Findings from a national quality improvement program in Australia. Social science \& medicine (1982). 2015 Nov;145:1-6. PMID: 26432175. doi: 10.1016/j.socscimed.2015.09.019.

19. Pratt NL, Kalisch Ellett LM, Sluggett JK, Gadzhanova SV, Ramsay EN, Kerr M, et al. Use of proton pump inhibitors among older Australians: national quality improvement programmes have led to sustained practice change. International journal for quality in health care: journal of the International Society for Quality in Health Care. 2017 Feb 1;29(1):75-82. PMID: 27920248. doi:

10.1093/intqhc/mzw138.

20. Kalisch Ellett LM, Lim R, Pratt NL, Kerr M, Ramsay EN, LeBlanc TV, et al. Reducing hypnotic use in insomnia management among Australian veterans: results from repeated national interventions. BMC health services research. 2018 August 09;18(1):626. doi: 10.1186/s12913-018-3443-9.

21. European Centre for Disease Control and Prevention. Coronavirus disease 2019 (COVID-19) pandemic: increased transmission in the EU/EEA and the UK - seventh update, 25 March 2020. Stockholm: ECDC. 2020. 2020: Available from: https://www.ecdc.europa.eu/sites/default/files/documents/RRA-seventh-update-Outbreak-ofcoronavirus-disease-COVID-19.pdf [Accessed 2 April 2020].

22. Guan W, Ni Z, Hu Y, Liang W, Ou C, He J, et al. Clinical Characteristics of Coronavirus Disease 2019 in China. New England Journal of Medicine. 2020 (e-pub ahead of print):DOI:

10.1056/NEJMoa2002032. 
23. Wang D, Hu B, Hu C. Clinical Characteristics of 138 Hospitalized Patients With 2019 Novel Coronavirus-Infected Pneumonia in Wuhan, China. JAMA. 2020;323(11):1061-9. doi:10.01/jama.2020.1585.

24. Zhou F, Yu T, Du R, Fan G, Liu Y, Liu Z, et al. Clinical course and risk factors for mortality of adult inpatients with COVID-19 in Wuhan, China: a retrospective cohort study. Lancet. 2020;395:1054-62. https://doi.org/10.16/S0140-6736(20)30566-3.

25. Wu Z, McGoogan J. Characteristics of and Important Lessons From the Coronavirus Disease 2019 (COVID-19) Outbreak in China. Summary of a Report of 72314 Cases From the Chinese Center for Disease Control and Prevention. JAMA. 2020. doi:10.1001/jama.2020.648. (e-pub ahead of print :.

26. Chen T, Wu D, Chen H, Yan W, Yang D, Chen G, et al. Clinical characteristics of 113 deceased patients with coronavirus disease 2019: retrospective study. BMJ. 2020;363:doi. https://doi.org/10.1136/bmj.m091.

27. CDC COVID-19 Response Team. Preliminary Estimates of the Prevalence of Selected Underlying Health Conditions Among Patients with Coronavirus Disease 2019 - United States, February 12March 28, 2020. Centers for Disease Control and Prevention Morbidity and Mortality Weekly Report. March 31 2020;Early Release / Vol 69:Available from: https://www.cdc.gov/mmwr/volumes/69/wr/pdfs/mm6913e2-H.pdf [Accessed April 2 2020].

28. Guan W, Liang W, Zhao Y, Liang H, Chen Z, Li Y, et al. Comorbidity and its impact on 1590 patients with Covid-19 in China: A Nationwide Analysis European Respiratory Journal. 2020:DOI: 10.1183/13993003.00547-2020.

29. Yang $X, Y u$ Y, Xu J, Shu H, Xia J, Liu H, et al. Clinical course and outcomes of critically ill patients with SARS-CoV-2 pneumonia in Wuhan, China: a single-centered, retrospective, observational study. Lancet Respiratory Medicine. 2020. https://doi.org/10.1016/S2213-600(20)30079-5. (e pub ahead of print).

30. Verity R, Okell L, Dorigatti I, Winskill P, Whittaker C, Imai N, et al. Estimates of the severity of coronavirus disease 2019: a model-based analysis. Lancet Infectious Diseases. 2020:DOI:https://doi.org/10.1016/S473-3099(20)30243-7.

31. Liang W, Guan W, Chen R, Wang W, Li J, Xu K, et al. Cancer patients in SARS-CoV-2 infection: a nationwide analysis in China. Lancet Oncology. 2020;21(3):335-7.

32. Greenhalgh T, Robert G, Macfarlane F, Bate P, Kyriakidou O. Diffusion of innovations in service organizations: systematic review and recommendations. Milbank Q. 2004;82(4):581-629. doi:10.1111/j.0887-378X.2004.00325.x. PMID: 15595944.

33. Veterans' MATES. Keeping well during the Coronavirus (COVID-19) pandemic: Three practical things you can do. 2020; Available from: https://www.veteransmates.net.au/topic-59-veterans-advice.

34. Davidson-Pilon C, Kalderstam J, Jacobson N, Reed S, Kuhn B, Zivich P, et al. CamDavidsonPilon/lifelines: v0.25.11. v0.25.11 ed. Zenodo; 2021.

35. Tomio J, Sato H. Emergency and disaster preparedness for chronically ill patients: a review of recommendations. Open Access Emerg Med. 2014;6:69-79. doi:10.2147/OAEM.S48532. PMID: 
27147882.

36. Slama S, Kim H-J, Roglic G, Boulle P, Hering H, Varghese C, et al. Care of non-communicable diseases in emergencies. The Lancet. 2017;389(10066):326-30. doi:10.1016/S0140-6736(16)31404-0.

37. Man RX-G, Lack DA, Wyatt CE, Murray V. The effect of natural disasters on cancer care: a systematic review. The Lancet Oncology. 2018 2018/09/01/;19(9):e482-e99. doi:

https://doi.org/10.1016/S1470-2045(18)30412-1.

38. Kopp JB, Ball LK, Cohen A, Kenney RJ, Lempert KD, Miller PE, et al. Kidney Patient Care in Disasters: Lessons from the Hurricanes and Earthquake of 2005. Clin J Am Soc Nephrol. 2007;2(4):814-24. doi:10.2215/cjn.03481006.

\section{Tables}

Table 1 - Baseline comparison between digital and post group 


\begin{tabular}{|lll|}
\hline & Digital & Post \\
\hline Total veteran participants & 51052 & 26859 \\
\hline Gender - Female (\%) & $24536(48.1 \%)$ & $12167(45.3 \%)$ \\
\hline Average age (SD) & $83.96(9.24)$ & $83.52(9.34)$ \\
\hline Living in community setting (\%) & $45759(89.6 \%)$ & $23113(86.1 \%)$ \\
\hline State (\%) & & \\
\hline NSW & $17758(34.8 \%)$ & $9626(35.8 \%)$ \\
\hline QLD & $10723(21 \%)$ & $6626(24.7 \%)$ \\
\hline VIC & $10647(20.9 \%)$ & $4905(18.3 \%)$ \\
\hline WA & $5293(10.4 \%)$ & $2004(7.5 \%)$ \\
\hline SA & $4260(8.3 \%)$ & $1731(6.4 \%)$ \\
\hline ACT & $1289(2.5 \%)$ & $1348(5 \%)$ \\
\hline TAS & $980(1.9 \%)$ & $468(1.7 \%)$ \\
\hline NT & $102(0.2 \%)$ & $151(0.6 \%)$ \\
\hline Number of comorbidities (\%) & & \\
\hline $\mathbf{1}$ & $18243(35.7 \%)$ & $9772(36.4 \%)$ \\
\hline $\mathbf{2}$ & $16820(32.9 \%)$ & $8980(33.4 \%)$ \\
\hline $\mathbf{3}$ & $10139(19.9 \%)$ & $5062(18.8 \%)$ \\
\hline $\mathbf{4}$ & $4166(8.2 \%)$ & $2202(8.2 \%)$ \\
\hline $\mathbf{5}$ & $10.24(8.03)$ & $8.13(8.64)^{\star}$ \\
\hline Average number of appointments with primary GP in $2019(\mathrm{SD})$ & $682(2.5 \%)$ \\
\hline
\end{tabular}

${ }^{*} p<0.01$

Table 2 - Coefficients of the Cox model 


\begin{tabular}{lll}
\hline Covariate & Hazard Ratio (Cl 95\%) & p value \\
\hline Medium (Post = 1) & $0.73(0.71,0.74)$ & $<0.005$ * \\
\hline Number of appointments in 2019 & $1.04(1.04,1.04)$ & $<0.005$ * \\
\hline Number of comorbidities & $1.09(1.08,1.10)$ & $<0.005 *$ \\
\hline Gender (Female = 1) & $1.05(1.03,1.08)$ & $<0.005$ *
\end{tabular}

${ }^{*} p<0.01$

Figures 


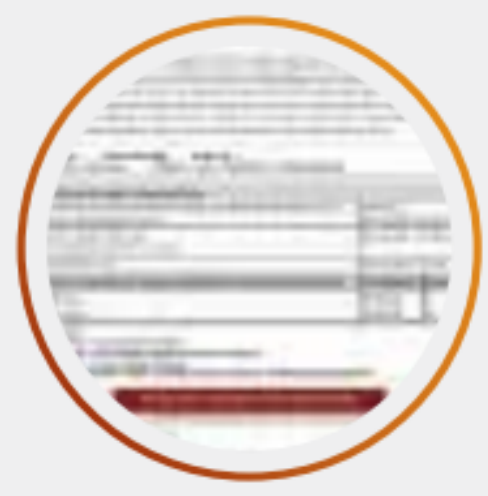

Electronic version of Patient Specific Feedback incorporating access to supporting therapeutic educational material is encrypted

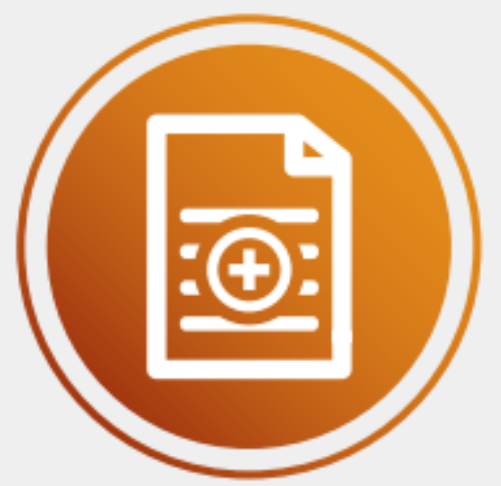

Health claims data used to generate patient specific feedback

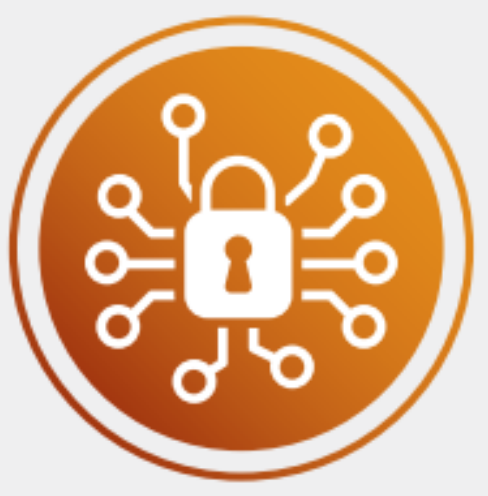

\section{Encrypted} message is forwarded to GP

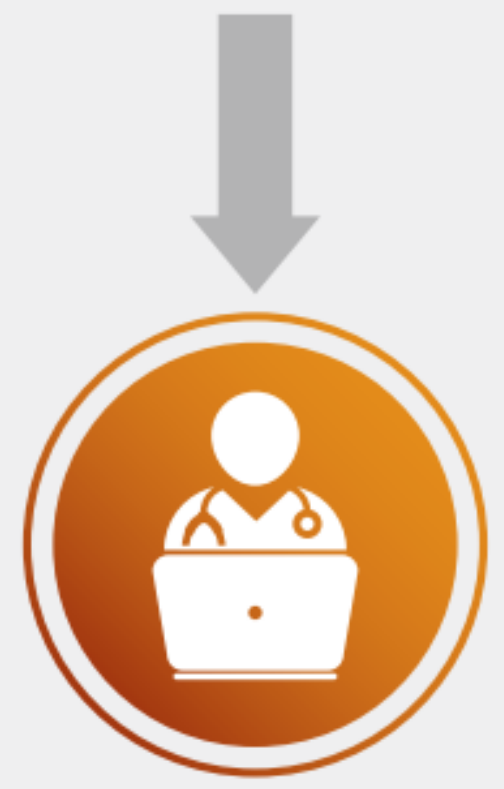

The message is downloaded into the GPs electronic medical record (EMR) system

Figure 1

Schematic representation of the digital intervention delivery (created by the authors) 
This Veterans' MATES information identifies your DVA clients who are at high risk of poor outcomes if they contract COVID-19. The risk factors for poor outcomes include older age, hypertension, chronic heart disease, diabetes, chronic airways disease, cerebrovascular disease, chronic liver disease, chronic renal failure, malignancy, and being immunocompromised or taking immune suppressing medicines.

You can access the summarised evidence on risk factors by clicking on covid factsheets There, you will also find up-to-date information about medicine use during the COVID-19 pandemic.

\section{FIRST \& SURNAME* DOB: $<D D / M M / Y Y Y Y>$ GENDER: <Male or Female> ACCOMMODATION: <Community or RACF} ADDRESS:

Total number of risk factors in addition to older age: 3

\begin{tabular}{|c|c|c|}
\hline Potential risk factors for poor outcomes with COVID-19 & $\begin{array}{c}\text { Last hospital admission } \\
\text { or service }\end{array}$ & $\begin{array}{l}\text { Last medicine } \\
\text { dispensing }\end{array}$ \\
\hline Hypertensive heart disease $\mathrm{e}^{1}$ or hypertension ${ }^{2}$ & - & 1 Feb 2020 \\
\hline $\begin{array}{l}\text { Chronic obstructive pulmonary disease }{ }^{1,2} \text {, asthma }{ }^{1,2} \text { or previous hospital admission } \\
\text { for pneumonia or influenza }{ }^{1}\end{array}$ & - & 1 Feb 2020 \\
\hline $\begin{array}{l}\text { Recent cancer treatment, including hos pital admission }{ }^{1} \text {, radiotherapy } y^{1,3} \text { or cytotoxic } \\
\text { therapy } y^{1,2}\end{array}$ & 27 Jan 2020 & - \\
\hline
\end{tabular}

1 morbidity identified through hospital record of diagnosis

2 morbidity identified through medicine claim for indication

3 morbidity identified through MaS claim for service

\section{Suggested actions:}

- Maintain contact with these vulnerable patients throughout the COVID-19 pandemic.

Ask your patients at high risk to contact your practice by phone if they develop respiratory symptoms. Ensure they are familiar with COVID-19 symptoms, what they can do to avoid contracting COVID-19 and who to contact if they are concerned. If you are caring for patients with COVID-19, closely monitor markers of clinical progression especially on days five to ten after onset of symptoms, the time point where rapid deterioration has frequently been observed.

- Schedule appointments to ensure vulnerable patients are still receiving necessary care. Discuss the options of telehealth and face-to-face consultations and identify the most appropriate option for you and your patient. Confirm their understanding of telehealth services, their preferred mechanism (e.g. telephone or video service) and their capability to participate in video telehealth services with you and their other health providers.

- Administer flu and pneumococcal vaccinations, where the patient is unvaccinated or a further dose of Pneumovax is required.

The Australian Therapeutic Goods Administration advises that the adjuvanted quadrivalent influenza vaccine, Fluad Quad, is preferred in persons aged 65 years and over and is available through the National Immunisation Program (NIP) Schedule.

Along with this letter, you will receive information about 4 other DVA clients. We appreciate the immense pressure GPs of Australia are currently experiencing and hope we can help support your care of DVA clients at this time.

*Hospital admissions identified in claims data in the past five years. Medicine use identified in PBS claims, with the patient having at least two claims for a medicine in this class in the past year. Most recent claim date for each service is shown in the table. Patient specific information is based on claims to DVA from all healthcare providers. Some of the medicines listed might have been prescribed by other doctors. You have been identified as the general practitioner who has written most of the recent prescriptions for this patient.

This information has been endorsed by the DVA Editorial Committee, which includes representatives from the AMA and RACGP.

For general comments and feedback please contact MATES.comments@ unisa.edu.au

\section{Figure 2} authors) 


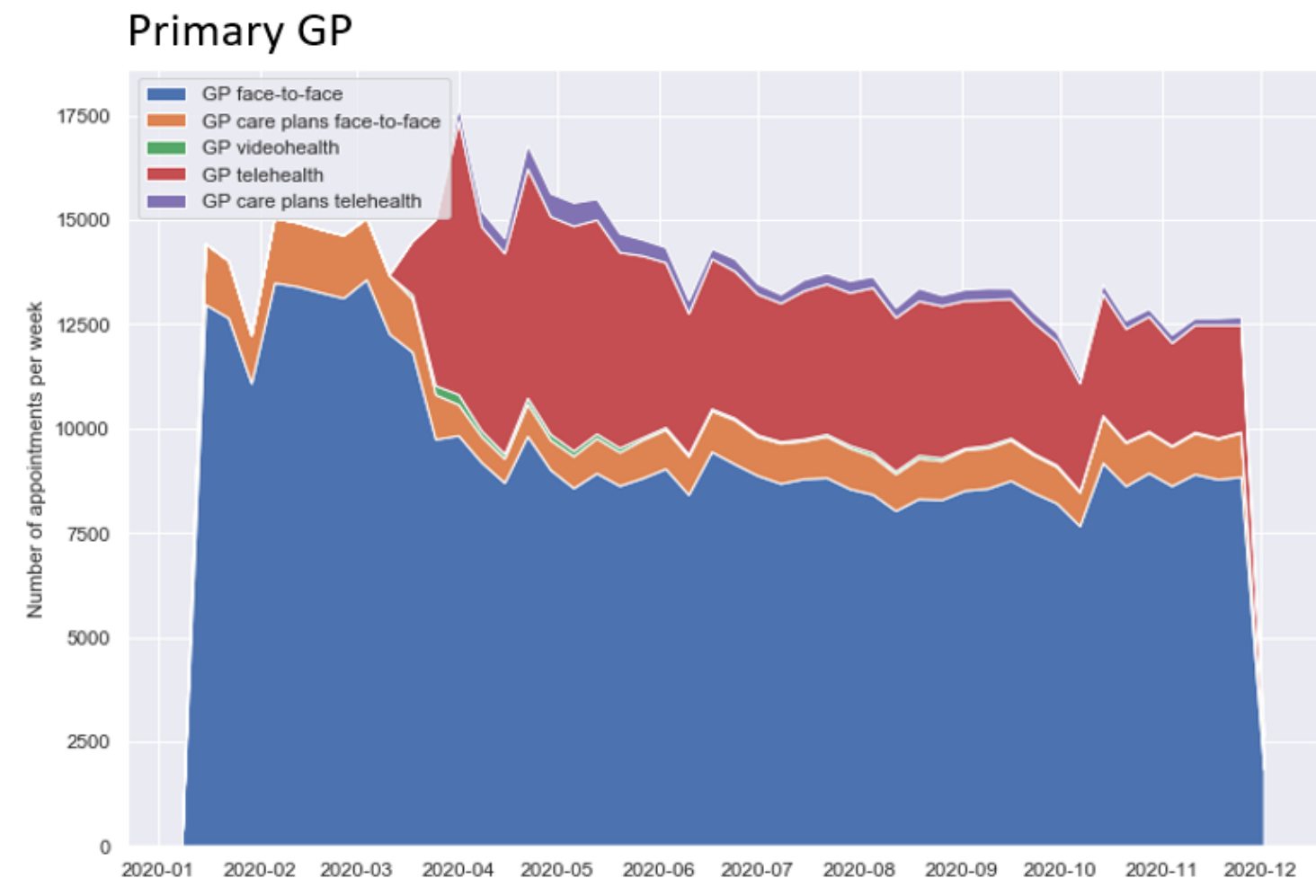

\section{Other than primary GP}

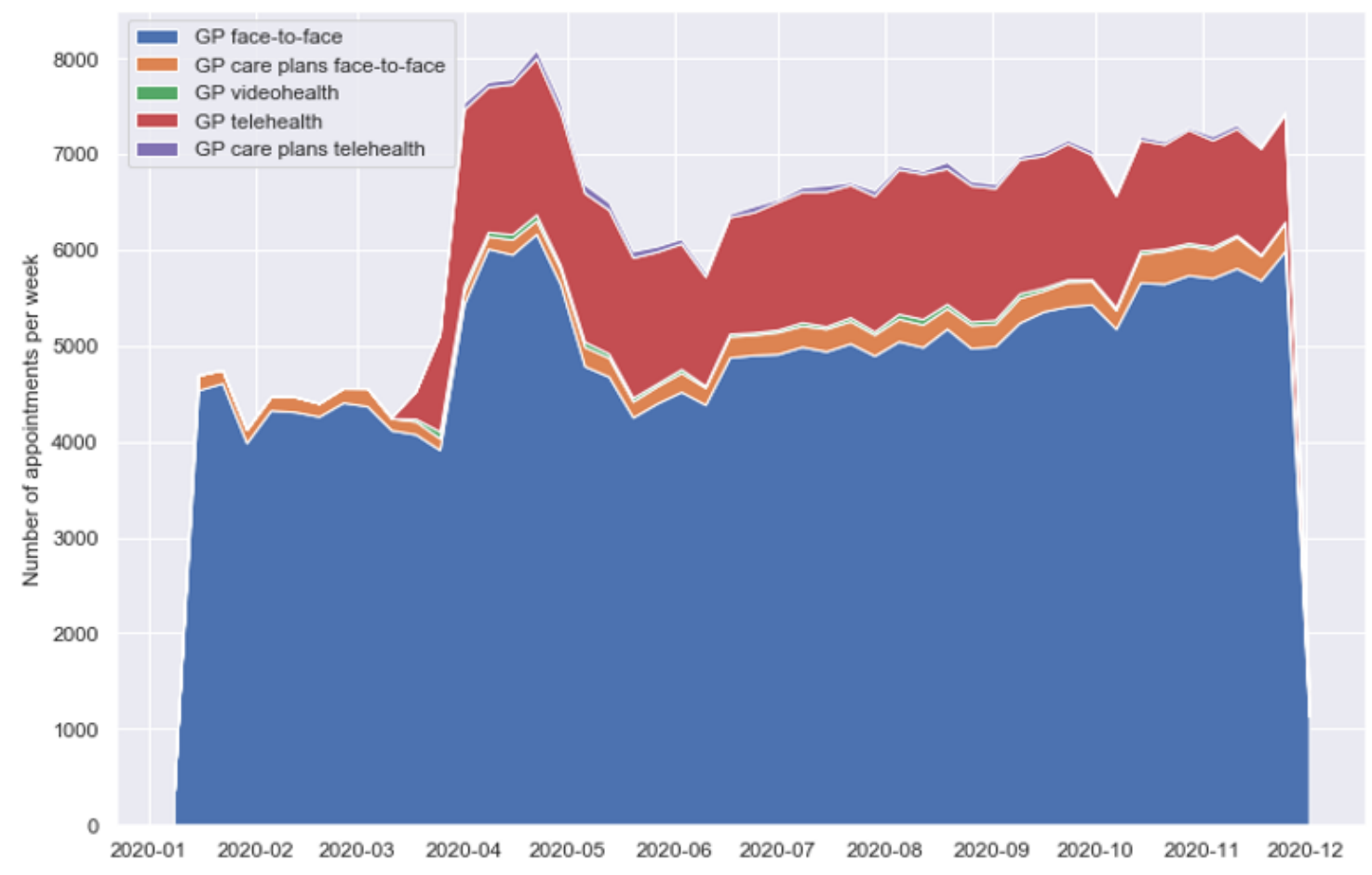

\section{Figure 3}

GP appointments during the first months of the COVID-19 pandemic, by type (created by the authors) 


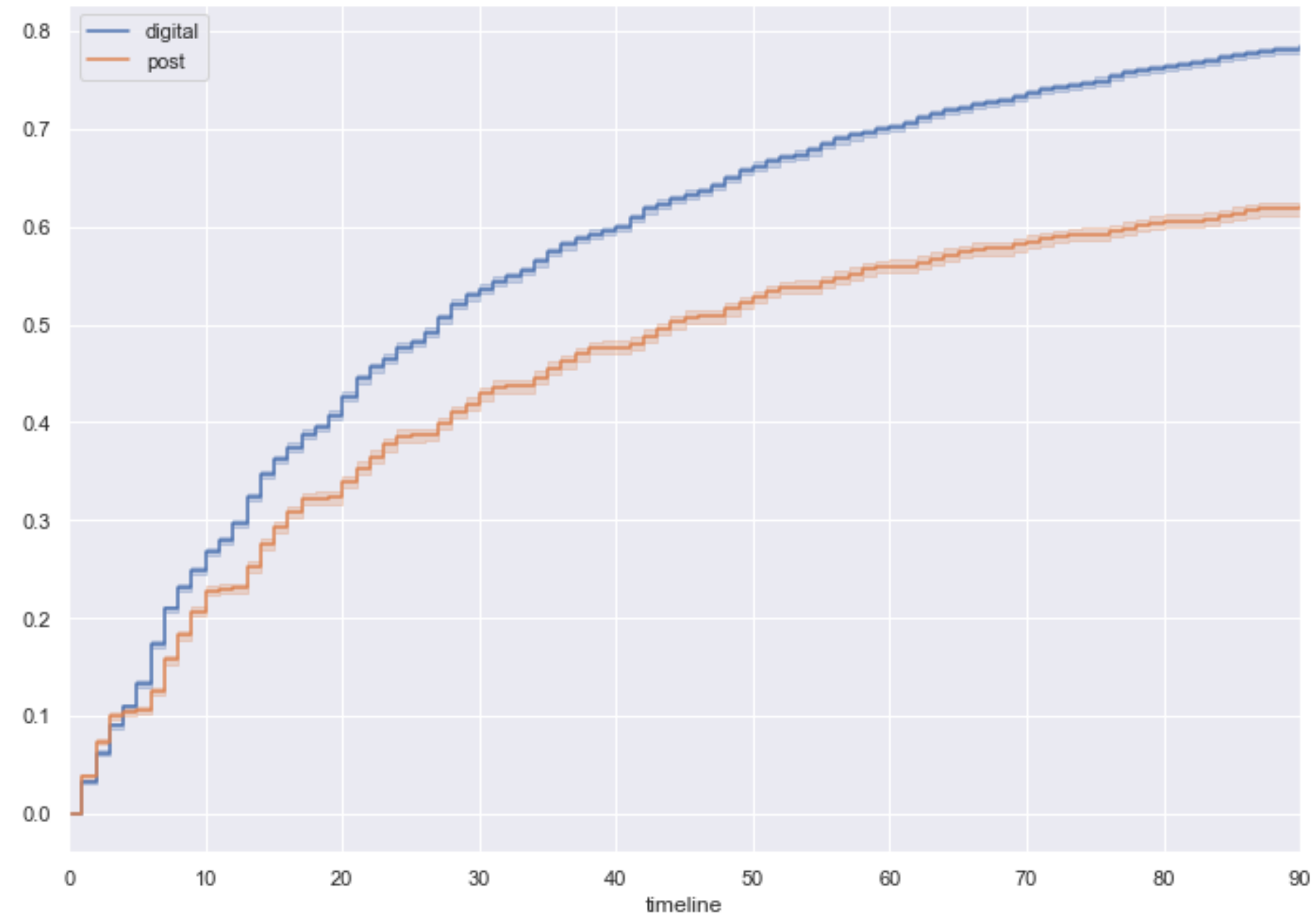

Figure 4

Kaplan Meyer curve depicting the chance of GP appointment in the first 3 months after intervention, by group (created by the authors) 


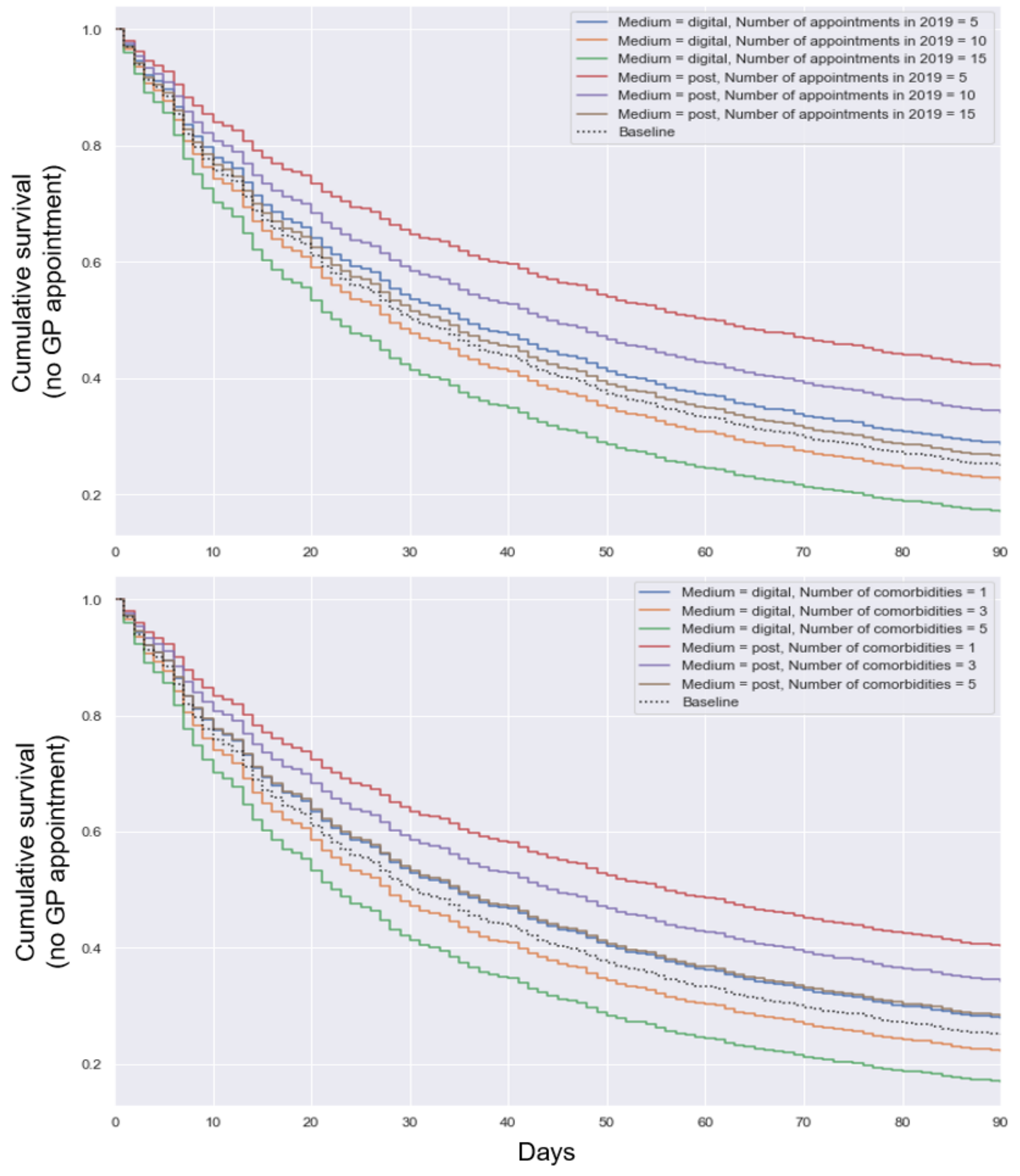

\section{Figure 5}

Partial effects from group (digital vs post), number of appointments and number of comorbidities on the chance of being left unassisted by the primary provider during the three months following intervention 